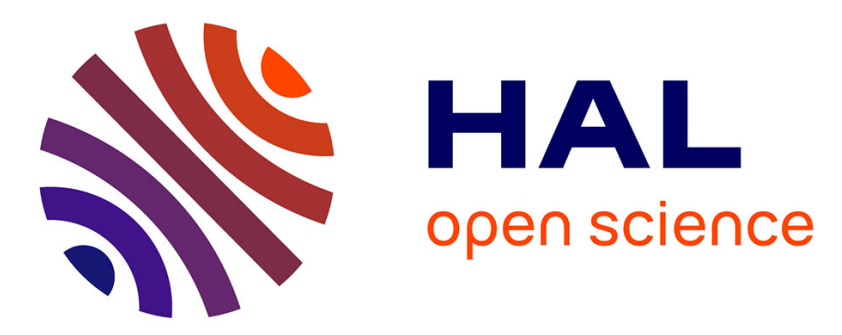

\title{
Quelques implications pédagogiques de l'apprentissage de l'arabe comme langue pluriglossique et langue de spécialité dans un enseignement militaire de type Lansad
} Marie Baize-Varin

\section{- To cite this version: \\ Marie Baize-Varin. Quelques implications pédagogiques de l'apprentissage de l'arabe comme langue pluriglossique et langue de spécialité dans un enseignement militaire de type Lansad. Recherche et pratiques pédagogiques en langues de spécialité - Cahiers de l'APLIUT, 2016, 35 ( $\mathrm{N}^{\circ}$ spécial 1), 10.4000/apliut.5561 . hal-02188499}

\section{HAL Id: hal-02188499 \\ https://hal.univ-rennes2.fr/hal-02188499}

Submitted on 18 Jul 2019

HAL is a multi-disciplinary open access archive for the deposit and dissemination of scientific research documents, whether they are published or not. The documents may come from teaching and research institutions in France or abroad, or from public or private research centers.
L'archive ouverte pluridisciplinaire HAL, est destinée au dépôt et à la diffusion de documents scientifiques de niveau recherche, publiés ou non, émanant des établissements d'enseignement et de recherche français ou étrangers, des laboratoires publics ou privés. 
Cahiers de l'Apliut

Vol. $35 \mathrm{~N}^{\circ}$ spécial 1 | 2016

Numéro spécial 1 - Du secteur Lansad et des langues

de spécialité

\section{Quelques implications pédagogiques de l'apprentissage de l'arabe comme langue pluriglossique et langue de spécialité dans un enseignement militaire de type Lansad}

A Few Pedagogical Implications on the Learning of Arabic as a Pluriglossic

Language for Specific Purposes in Military Fields and Military Teaching to

Cadets

Marie Baize-Varin

\section{OpenEdition}

Journals

Édition électronique

URL : http://journals.openedition.org/apliut/5561

DOI : 10.4000/apliut.5561

ISSN : 2119-5242

Éditeur

APLIUT

Ce document vous est offert par Université Rennes 2

Référence électronique

Marie Baize-Varin, «Quelques implications pédagogiques de l'apprentissage de l'arabe comme langue pluriglossique et langue de spécialité dans un enseignement militaire de type Lansad », Recherche et pratiques pédagogiques en langues de spécialité [En ligne], Vol. 35 Nº spécial 1 | 2016, mis en ligne le 30 octobre 2016, consulté le 18 juillet 2019. URL : http://journals.openedition.org/apliut/5561; DOI : 10.4000/apliut.5561

Ce document a été généré automatiquement le 18 juillet 2019. 


\title{
Quelques implications pédagogiques de l'apprentissage de l'arabe comme langue pluriglossique et langue de spécialité dans un enseignement militaire de type Lansad
}

\author{
A Few Pedagogical Implications on the Learning of Arabic as a Pluriglossic \\ Language for Specific Purposes in Military Fields and Military Teaching to \\ Cadets
}

Marie Baize-Varin

\section{Pluriglossie et langue de spécialité : essai de redéfinition de quelques termes}

Avant d'aborder la question de l'enseignement de la langue arabe comme langue de spécialité, il est nécessaire d'en définir la spécificité en tant que langue pluriglossique, c'est-à-dire comprenant plusieurs glosses ou variétés. L'arabe militaire que nous enseignons est souvent vu comme une langue de spécialité ressortissant de la glosse arabe classique. Nous montrerons dans cet article que la réalité de l'usage des arabophones ne reflète pas systématiquement ces classifications. Un essai préalable de redéfinition des différents termes utilisés dans l'étude de l'arabe comme langue pluriglossique, s'avère ici nécessaire.

2 Il est donc souvent dit dans le milieu des arabisants que l'arabe est une langue diglossique. Cette classification est présentée ainsi par Larcher («Diglossie arabisante »1) :

Jusqu'à date très récente, les arabisants se faisaient une représentation de l'arabe résumée par un maître-mot, celui de diglossie, c'est-à-dire la coexistence de deux 
variétés d'une même langue : l'une appelée classique, littéraire ou littérale (angl.Classical Arabic) vue à l'origine de l'arabe moderne (angl. Modern Standard Arabic), et l'autre appelée dialectale (angl. colloquial), abstraction désignant la classe des dialectes.

Selon le même auteur (Larcher «Diglossie arabisante»1) cependant «l'adéquation descriptive du concept de diglossie, s'agissant de la situation linguistique de l'arabe » est de plus en plus contestée chez les arabisants, même si certains la soutiennent encore (Rosenbaum 68, note 2). Larcher conclut d'ailleurs son article « Diglossie arabisante » par l'assertion suivante, résumant leur position actuelle :

[...] les arabisants ont décliné diglossie d'abord en triglossie, puis, la troisième glosse étant soit l'arabe moderne, soit l'arabe moyen, en quadri- ou tétraglossie et enfin en multi-, pluri- ou polyglossie. (12)

La pluriglossie de l'arabe est définie ainsi par Dichy («La pluriglossie en (inter)action)») :

Le concept de pluriglossie reflète notamment le fait que ce système complexe correspond à une seule et même langue constituée d'un ensemble de variétés ou glosses incluses dans la compétence communicative du locuteur : ce dernier fait choix de la glosse appropriée - ou apprécie celle de ses interlocuteurs - en fonction de la situation de communication [...], mais également de contraintes qui relèvent de l'articulation générale du vocabulaire au sein de la pluriglossie [...]. (2)

Si le concept de diglossie, avant d'être appliqué à l'arabe, lui était exogène (Larcher «Diglossie arabisante »), en est-il de même pour le concept de pluriglossie ? Selon l'auteur (Larcher « Diglossie arabisante » 10), une première conception «triglossique » de l'arabe reviendrait à Ibn Khaldoun (Ibn Hुaldūn, m. 1406), qui distingua, dans sa Muqaddima (ch. 47, 1075), un type d'arabe du Nord des dialectes bédouins et des dialectes citadins. À l'issue de cet historique du concept de diglossie chez les arabisants, l'auteur (Larcher «Diglossie arabisante »12) conclut en expliquant le fait que «le monde arabe s'est rallié à la représentation diglossique de l'arabe, au moment même où les arabisants l'abandonnent ».

6 Même si notre article ne traite que de deux variétés de l'arabe, artificiellement séparées dans notre pratique pédagogique pour les raisons expliquées infra, nous restons persuadée que l'arabe n'est pas une langue diglossique. Elle est bien pluriglossique si l'on se base sur le continuum des différentes glosses en jeu en synchronie dans la compétence de communication d'un locuteur arabe scolarisé (Dichy «La pluriglossie en (inter)action »).

7 Une présentation générale de cette pluriglossie a été faite par Dichy ( « L'enseignement de l'arabe »3) dans le cadre du choix de l'arabe à enseigner dans le secteur public français, et par le même auteur (Dichy «La pluriglossie en (inter)action » 2) dans la compétence de communication d'un locuteur arabophone scolarisé. Dichy («L'enseignement de l'arabe » 3) ajoute aux dialectes arabes ${ }^{1}$, « vecteurs des cultures locales», ce qu'il appelle «l'arabe littéraire», « vecteur de la culture commune à l'ensemble des pays arabes». Si Dichy («L'enseignement de l'arabe » 3) prend « littéraire » dans son sens général, nous cautionnons cet emploi. S'il emploie « littéraire" stricto sensu, il nous semble que cette définition est restrictive. En effet, « l'arabe littéraire » n'est pas cantonné au domaine de la littérature mais bien utilisé dans des domaines aussi divers que la politique, l'économie, les finances, les sciences, les médias etc.

8 Il se peut aussi que Dichy («L'enseignement de l'arabe » 3) utilise " littéraire » dans un sens ancien qui pourrait correspondre à une variété prestigieuse de langue, donc 
rattachée à la littérature. Cette réflexion nous vient à la lecture de Larcher («Diglossie arabisante »4) quand il précise qu'en 1795, à la fondation de l'École des langues orientales, un article du décret de la fondation de cet établissement «stipule que celle-ci 'sera composée d'un professeur d'arabe littéraire et vulgaire' [...]». Dichy («La pluriglossie en (inter)action » 1 , note 1) précise d'ailleurs que « littéraire » relève "d'un usage archaïsant du français » sans donner plus d'indication sur le contenu sémantique de cet usage, avant d'ajouter que "[l]'expression arabe littéraire est utilisée par les francophones du Moyen-Orient comme équivalent de al-'arabiyya l-fushâ ». Il s'agit bien ici d'un équivalent, et non pas d'une traduction car al-'arabiyya l-fushâ signifie "l'arabe le plus éloquent ». Plus précisément, Larcher («L'arabe, plus qu'une langue?») explique ceci :

À l'origine, le terme de fasîh, dont fushâ est l'élatif (le correspondant de notre comparatif/superlatif) féminin ne qualifie pas la langue comme «pure », mais celui qui parle comme « éloquent ». (47)

Dichy («L'enseignement de l'arabe» 3 ) subdivise «l'arabe littéraire» en « un arabe littéraire classique (langue, pour faire court, des époques médiévales)» et "un arabe littéraire moderne ", faisant de "classique " un critère chronologique, ce qui n'est, à notre avis, que la résultante superficielle de la correspondance usuelle des sens " classique » et " ancien ». Larcher ("Qu'est-ce que l'arabe » 11) introduit un autre critère à " classique » que le critère chronologique : il en fait une "étiquette sociolinguistique », se basant sur l'étymologie du mot.

[...] la double extension de sens de classique, la première métaphorique (« de première classe ») et la seconde jouant sur la polysémie du mot «classe » («qui s'enseigne dans les classes »), lui convient parfaitement : l'arabe classique est bien tout à la fois la variété de prestige et la norme scolaire. (39)

Pour en revenir au critère chronologique, Larcher ("Qu'est-ce que l'arabe ») précise que «l'apparition d'une variété 'classique' est situable chronologiquement dans l'histoire d'une langue ", mais que :

[c]ontrairement à ce que croient beaucoup d'arabisants [...], l'arabe classique n'est pas l'état de l'arabe commençant vers $500 \mathrm{ap}$. JC, époque à laquelle apparaissent les premières inscriptions qui soient à la fois en arabe et en écriture arabe. (38)

11 Ainsi donc, pour nommer la variété d'arabe que Dichy («L'enseignement de l'arabe » 3) nomme «littéraire », nous préférons "classique » (langue de première classe et langue enseignée dans les classes, à l'école), qui nous semble correspondre davantage à sa réalité linguistique et sociolinguistique, à « littéraire » au sens strict du terme, pour les raisons invoquées supra. Il arrive que l'arabe classique soit aussi nommé « littéral », c'est-à-dire " écrit », ce qui ne correspond pas non plus complètement à sa réalité, puisqu'il peut être également parlé. Certains l'appellent également «standard" pour le distinguer des dialectes arabes et sous-entendre qu'il est unifié du Maroc à l'Irak, ce que nous ne cautionnons pas non plus, étant donné les variations que l'on peut y constater d'un pays à l'autre et sur lesquelles nous ne nous étendrons pas.

Dichy («L'enseignement de l'arabe » 3-4) décrit la « compétence de communication d'un locuteur arabe scolarisé » comme comprenant donc l'arabe dialectal, ainsi que «l'arabe littéraire, dans ses deux états classique et moderne ». Nous appellerons ces derniers pour notre part « l'arabe classique ancien » et « l'arabe classique moderne », préférant pour les raisons expliquées supra la classification de Larcher, même si celle de Dichy reflète le plus ancien usage chez les arabisants ${ }^{2}$. 
13 Nos choix de termes pour désigner les différentes variétés d'arabe étant exposés, venonsen à présent au concept de langue de spécialité, tel que défini par Spillner cité par Messaoudi qui aborde dans son article la question de l'arabe comme telle. Selon Messaoudi (128), Spillner pose la langue de spécialité comme étant constituée "par l'ensemble des éléments linguistiques qui peuvent se manifester, dans une situation donnée, lors de la communication entre spécialistes d'une même discipline ». Concernant l'arabe militaire, peut-on alors considérer que cette spécialité n'est que lexicale comme a tendance à le présenter al-Hakkak (5-6) ? Comme le demande Messaoudi (2010:127) dans son article qui a le mérite de poser méthodologiquement le problème, «les langues spécialisées possèdent-elles véritablement un fonctionnement propre, différent de celui de la langue générale? », entendant vraisemblablement par « fonctionnement propre » le fonctionnement syntaxique?

14 Au-delà de l'arabe militaire, le concept de langue de spécialité a-t-il été défini pour l'arabe en général ? S'agit-il d'un arabe lexicalement spécialisé, syntaxiquement spécialisé, ou les deux ? Lelubre définit quatre composantes [qui] contribuent à l'établissement du texte de spécialité :

- la composante terminologique : la terminologie de la spécialité concernée, c'est-àdire les dénominations des unités référentielles constituant le domaine traité ;

- la composante phraséologique : les phraséologies relatives à cette terminologie, c'est-à-dire les contextes préférentiels d'occurrence des termes dans les textes de spécialité ;

la composante brachygraphique : les signes brachygraphiques ${ }^{3}$;

- la composante discursive:les expressions propres aux types de discours intervenant dans les textes de la spécialité concernée. (163-164)

Puisque qu'il est question d'arabe militaire, abordons à présent les aspects de l'enseignement de cette langue à Saint-Cyr, et comment s'articulent langue de spécialité militaire et langue pluriglossique dans ce contexte.

\section{La question de l'enseignement du dialecte à l'École Spéciale Militaire}

Ce que les milieux militaires appellent communément « Saint-Cyr Coëtquidan » est une grande école sous tutelle du ministère de la Défense, exclusivement destinée à la formation initiale militaire, académique, et comportementale des élèves - officiers et officiers - élèves de l'armée de Terre française. L'enseignement de l'arabe y est particulièrement révélateur de la pluriglossie de cette langue, enseignée également en tant que langue de spécialité militaire.

17 En effet, l'arabe est d'une part étudié par les élèves de l'École spéciale militaire (ESM), nommée "Saint-Cyr » en référence à l'agglomération dans laquelle cette école est née avant d'être transplantée en Bretagne par le général de Gaulle suite à la Libération. Cette école forme des élèves ayant le niveau bac +2 après une classe préparatoire aux grandes écoles civile ou militaire. Les élèves de l'ESM sortent sous-lieutenants (officiers-élèves) à l'issue d'une formation intégrée militaire, académique et comportementale de trois ans, repartie en trois semestres militaires encadrant deux semestres académiques et un semestre à l'étranger. Les arabisants des deux filières Relations internationales et stratégie (RIS) et Management des hommes et des organisations (MHO) apprennent l'arabe syro-libanais depuis 2003. 

classique en lycée militaire et qui continuaient à apprendre cette langue - toujours dans sa variété classique - à Coëtquidan, réussissaient rarement à obtenir le certificat militaire de pratique parlée élémentaire (CMPPE), obtenu avec une moyenne comprise entre 8 et $10 / 20$, ou le certificat militaire de pratique écrite élémentaire (CMPEE). Ils réussissaient encore moins le certificat militaire de langue écrit du premier degré (CMLE1) ou le Certificat militaire de langue parlé du premier degré (CMLP1) malgré tous les efforts fournis. arabe-français/français-arabe étaient, et sont toujours autorisés dans les examens écrits organisés par le Cfiar, ce qui incitait les élèves à ne pas faire l'effort nécessaire pour apprendre le vocabulaire fondamental de base. En outre, l'utilisation du dictionnaire arabe, pour être un tant soit peu efficace, doit être précédée d'un apprentissage systématique et exhaustif des schèmes morphologiques et lexicaux. Le dictionnaire qui devrait être un outil de consultation ultime et de vérification, devient alors la cause même de l'échec de l'apprentissage de l'arabe, car les élèves comptent sur lui alors même qu'ils ne savent pas s'en servir.

23 Le problème, en outre, est que ces dictionnaires sont en général les éditions les plus récentes du Sabil Larousse de Reig, lequel ne rend pas compte, même dans l'édition de 2011, des néologismes que l'on rencontre en arabe de presse, comme par exemple 'intihârî dans son emploi apparemment substantivé, qui désigne un « auteur d'un attentat-suicide (communément nommé « kamikaze ») » (Reig 678). Ceci est peut-être dû au fait que, selon une supposition de Franjié, les médias arabes utilisent la plupart du temps les termes chahîd ou tafjîrî, et que 'intihârî dans le sens de " auteur d'un attentat-suicide » est une projection occidentale résultant de la référence au mode opératoire des kamikazes japonais ${ }^{4}$. 

égyptien - et, plus précisément du Caire - l'objet de l'enseignement de l'arabe aux SaintCyriens, et ce du fait du nombre des locuteurs de ce dialecte, ainsi que de l'importance de son impact historique et socioculturel (films, programmes et séries télévisées, livres, théâtre, chants et musique, Le Caire étant le siège de la Ligue arabe). C'est un des dialectes les plus répandus et les mieux compris dans une situation de communication entre arabophones de pays différents. De plus, la sous-direction de la formation et des écoles, directement subordonnée à la Direction des ressources humaines de l'armée de Terre, et qui s'occupe de tous les organismes de formation de cette armée, avait demandé à ce que l'accent soit mis sur « la pratique de la langue courante » pour les Saint-Cyriens. Le choix de l'égyptien n'est pas pour autant exclusif car la connaissance de ce seul dialecte permet très vite, après quelques temps d'adaptation, de comprendre les autres dialectes. Enfin, il est beaucoup plus facile d'atteindre un niveau moyen et dans des délais raisonnables en arabe dialectal qu'en arabe classique, l'accent étant mis sur l'oral dès le départ au détriment de l'apprentissage du système graphique, le but final étant de savoir commenter une image en quinze minutes au moins pour le CMLP1. Ceci implique l'utilisation de la transcription phonétique comme outil d'apprentissage. de l'arabe classique, le premier étant également une véritable langue avec un système grammatical et lexical que l'on peut enseigner et systématiser par des grammaires, des manuels et des dictionnaires. Mais les arabisants savent que l'apprentissage de l'alphabet arabe - alors que l'on peut utiliser la transcription phonétique pour le dialecte ${ }^{8}-$, ainsi que de la lecture prennent du temps et peuvent retarder un passage immédiat à l'oral.

De plus, avec l'accord de notre commandant de division et depuis 2010, nous avons remplacé les examens écrits de fin de semestre par des examens oraux, qui servent de préparation au CMLP1. 


\subsection{Depuis 2003 : le syro - libanais}

29 En 2003, sur instruction du général Thorette, Chef d'état-major de l'armée de Terre, qui fut lui-même arabisant, nous avons remplacé l'égyptien par le syro-libanais, pour les mêmes raisons pédagogiques qui ont présidé à l'abandon de l'enseignement de l'arabe classique, et pour des raisons stratégiques, les théâtres d'opération actuels ne se situant pas en Egypte mais au Proche-Orient. Ce choix est également sociolinguistique, l'arabe syro-libanais étant, avec l'arabe égyptien, un des dialectes les mieux compris dans le monde arabe. En effet, la Syrie et surtout le Liban, inondent régulièrement ce dernier de leurs productions télévisuelles, musicales et cinématographiques, par le biais des chaînes satellitaires.

30 Les modalités d'organisation de l'enseignement et de l'évaluation restent les mêmes que pour l'égyptien, et n'ont pas changé jusqu'à aujourd'hui. De plus, il existe de plus grandes différences d'ordre pédagogique entre l'enseignement de l'arabe classique avec tout ce qu'il implique en terme d'apprentissage de l'écriture et des techniques de lecture, et l'enseignement d'un arabe dialectal, qu'entre l'enseignement de deux dialectes qui appartiennent respectivement à la même famille proche-orientale. L'appellation générique de syro-libanais cache le fait qu'il s'agit avant tout de la variante diatopique syrienne de ce dialecte, et plus précisément encore de la variante damascène ${ }^{9}$. Les dialectes arabes sont rarement uniformisés au niveau d'un pays et, dans le cadre des cours de dialecte, c'est essentiellement celui de la capitale qui est enseigné. Il n'en reste pas moins que le syro-libanais, parlé en Syrie, au Liban, dans les Territoires Palestiniens et en Jordanie, est le creuset de caractéristiques communes majeures.

Les élèves peuvent ainsi mieux communiquer en arabe après les cent cinquante heures d'enseignement prévues car ils auront pu apprendre immédiatement à parler sans avoir à passer du temps sur l'apprentissage de l'alphabet. Ceci motive davantage les débutants qui, en apprenant l'arabe classique, ont parfois l'impression de piétiner avant de commencer à s'exprimer. De plus, le fait d'apprendre l'arabe dialectal avant l'arabe classique met les élèves dans les mêmes conditions d'apprentissage que les Arabes et les arabophones eux-mêmes. Cette approche de l'enseignement permet un passage plus facile à l'arabe classique écrit et oral auquel une partie de l'enseignement est consacrée (apprentissage de l'écriture, lecture et compréhension de textes simples) en fin de parcours, c'est-à-dire après le passage du CMLE1 de syro-libanais en avril. Les cours continuant entre avril et juin, les arabisants n'ayant jamais fait d'arabe classique en apprennent les bases, tandis que les élèves qui l'ont déjà étudié en lycée militaire ou dans un autre cadre approfondissent les leurs, ce qui pose à l'enseignant le problème d'une pédagogie différenciée au sein du même groupe.

Le but de notre enseignement est donc de faire acquérir aux élèves, dans un laps de temps réduit et au sein d'un environnement non-arabophone, un maximum de réflexes d'expression orale. L'enjeu reste par la suite de réutiliser cette base dans des stratégies de reformulation et d'expression libre autour d'une image.

Le dialecte étant le registre de la langue générale du quotidien, quelle peut être son articulation pédagogique avec l'enseignement d'une langue dite de spécialité ? 


\subsection{Dialectes arabes et langue de spécialité} classification qu'en propose Lelubre (1995:163-164), mentionné supra. L'arabe militaire en tant que langue de spécialité se distinguerait donc de l'arabe général, en tant que langue spécifique lexicalement, phraséologiquement et discursivement. Se pose alors la question de la différence écrit-oral, qui recoupe d'ailleurs la séparation traditionnellement faite entre arabe classique et arabe dialectal. Bon nombre d'arabophones et d'arabisants limitent l'arabe classique au registre écrit par opposition au dialecte, langue parlée de tous les jours et, par là même, sous-entendent qu'on ne pourrait pas parler en langue de spécialité. Ceci revient à nier deux réalités. La première est que l'arabe classique, bien que n'étant la langue maternelle de personne, est aussi parlé dans des situations spécifiques (interactions entre deux arabophones scolarisés originaires de pays arabes différents, arabe parlé des médias nationaux et transnationaux, débats télévisés, etc.), et ne s'utilise pas uniquement sous forme d'écrit oralisé. La seconde réalité est qu'un arabophone non scolarisé peut, à l'oral et selon sa profession, atteindre un niveau de haute technicité (Messaoudi 133-134) et utiliser ce que Dichy ( « La pluriglossie en (inter)action » 2) nomme " arabe moyen de type 2 », à savoir l'insertion de syntagmes ressortissant de l'arabe classique dans des phrases à syntaxe dialectale.

(technolecte" qui aurait l'avantage de désigner un "ensemble langagier spécialisé, qu'il soit écrit ou oral ", un «ensemble d'usages lexicaux et discursifs, propres à une sphère de l'activité humaine ». Ce " technolecte » ne peut être limité à un lexique, contrairement à une terminologie ou un jargon, car il « englobe aussi des usages discursifs ». Contrairement à l'emploi de langue de spécialité, le technolecte ne suggérerait pas d'opposition avec la langue ordinaire, puisque contenant lui-même "le vocabulaire banalisé et la terminologie populaire ». Si nous retenons cette définition du technolecte, il nous semble qu'elle recoupe celle de langue : c'est peut-être là que se rejoignent, en ce qui nous concerne, les notions d'enseignement de type Lansad et l'enseignement de la langue de spécialité militaire. En effet, enseigner un dialecte arabe avec tout ce qu'il contient en termes de vocabulaire général n'empêche aucunement de l'enseigner également dans un cadre militaire, en le spécialisant lexicalement. Il ne sera spécialisé que lexicalement, la phraséologie militaire relevant de l'arabe classique.

est donc démontré que l'arabe dialectal peut être, si son locuteur fait preuve d'un degré de haute technicité, une langue de spécialité, du moins lexicalement. Syntaxiquement, la phraséologie est davantage empruntée à l'arabe classique, ce qui donne lieu à l'utilisation de l'arabe moyen de type 1 , mentionné supra.

\subsection{Une implication pédagogique de l'arabe dialectal de spécialité}

En cours de syro-libanais, nous proposons à nos élèves d'apprendre les structures de l'appel militaire dans la variante syrienne de l'arabe syro-libanais, nous basant sur les usages réels de l'armée syrienne ${ }^{10}$. Cette activité nous permet d'introduire chez nos élèves des réflexes d'expression orale issus de l'apprentissage par cœur, ainsi que de les entraîner à se présenter militairement en arabe devant leur examinateur de CMLP1.

Recherche et pratiques pédagogiques en langues de spécialité, Vol. 35 № spécial 1 | 2016 

n'ont pas besoin d'avoir un niveau élevé pour maîtriser cette dernière. En effet, ces structures, de par leur simplicité syntagmatique (impératifs, groupes nominaux, annexion arabe correspondant au syntagme nom+complément du nom), sont facilement assimilables par cœur sans avoir à être grammaticalement conceptualisées. Nous les enseignons d'ailleurs environ un mois après le début de l'apprentissage des élèves, durant leur premier semestre académique (après environ vingt heures de cours sur cinquantesix). Le problème se pose différemment quand il s'agit de maîtriser la phraséologie de l'arabe classique journalistique à tendance militaire, telle que nous l'enseignons à l'EMIA.

\section{Organisation de l'enseignement de l'arabe classique à l'EMIA}

\subsection{De 2009 à aujourd'hui}

En 2009, l'EMIA a été réformée dans le but de calquer sa scolarité sur celle de l'ESM, avec deux semestres de moins cependant. Cette réforme a précipité la fin des «scolarités spécifiques ", les enseignements délivrés sous convention avec des universités.

Le but de la réforme était d'élaborer une « licence EMIA » de type professionnel, obtenue en deux ans et approuvée par le Conseil national de l'enseignement supérieur et de la recherche. L'enseignement de l'arabe - auparavant inspiré du programme d'Aix-Marseille Université -s'est recentré sur le lexique militaire, l'apprentissage de la phraséologie militaire requérant un volume plus conséquent d'heures de cours. La plupart des élèves sont maintenant débutants mais nous disposons d'un nombre suffisant d'heures $(260 \mathrm{~h}$ sur un an et demi) pour continuer à leur enseigner les bases graphiques et grammaticales de l'arabe classique. 
Notre enseignement s'articule essentiellement autour de la préparation au CMLP1 d'arabe classique: le commentaire pendant quinze minutes au moins d'une image sur des questions de défense, suivi d'une discussion d'ordre général d'environ dix minutes. Les élèves réussissent en général assez bien cette épreuve puisque nous les habituons à parler dès le début des cours, même si l'alphabet n'est pas entièrement vu, en opérant un va-etvient constant entre l'oral à manier par cœur (formules de présentation etc.), et la reconnaissance des lettres qu'ils apprennent au fur et à mesure-d'abord en reconnaissance puis en reproduction - dans les mots qu'ils connaissent déjà. Nous les habituons dès le départ à la phraséologie de l'analyse d'image et des phrases descriptives et argumentatives, et nous voyons avec le recul de notre pratique, que cette répétition s'avère plus utile qu'une réflexion théorique sur la grammaire et le fonctionnement de la langue.

Il reste néanmoins le problème de ce que nous appelons l'absence « d'indexicalité » dans la phraséologie de nos élèves à la suite de Dressen-Hammouda.

\subsection{L'absence d'indexicalité en arabe classique dans le commentaire d'image}

5 Dressen-Hammouda nomme "indexicalité » la "socialisation langagière » : la capacité contextuelle et implicite à ne pas se limiter à la sémantique et la syntaxe d'une langue pour rendre correctement son sens dans un processus de traduction. Elle parle, concernant la langue anglaise, d'énoncés d'apprenants issus de la traduction du français vers l'anglais, et qui «ne font pas anglais » (je cite, 2015). Ces énoncés ne sont donc pas idiomatiques, tout en restant corrects d'un point de vue strictement linguistique. Les énoncés de nos élèves, malgré leur correction grammaticale, ne sont globalement pas idiomatiques. Au cours des dernières heures de préparation à l'analyse d'image, les élèves élaborent en petits groupes une production qu'ils nous soumettent par la suite oralement. Nous leur recommandons de penser directement en arabe et de choisir des énoncés simples, sans élaborer mentalement des phrases complexes en français, qu'ils auraient du mal à traduire par la suite.

Ils ne suivent pas systématiquement notre conseil : ils élaborent des énoncés simples mais traduits du français, ce qui donne la plupart du temps des énoncés dépourvus d'indexicalité, sachant qu'il y a une forte part de subjectivité dans ce que nous pouvons considérer comme idiomatique ou non-idiomatique. En effet, la phraséologie de l'arabe moderne de presse touchant aux questions militaires évoluant constamment du fait des traductions arabes de communiqués de presse en anglais ou français, de la formation à l'étranger des journalistes arabes etc, il est difficile pour nous et scientifiquement inapproprié de poser ce qui est idiomatique ou non en arabe. Dans notre pratique pédagogique, nous tentons néanmoins de développer une sensibilité particulière à la phraséologie de nos élèves.

Nous donnons ici un exemple d'énoncé fourni par un élève de l'EMIA début 2016 :

Wa-fî l-rasm al-thâlith al-'alam 'abyadh wa-'aswad, wa-bi-l-muhâdhât [traduction littérale de l'expression française ne correspondant pas à l'usage arabe] al-shakhs 'inda-hu 'asliha 'akthar fa-'akthar [traduction littérale de l'expression française, alors que la forme verbale dérivée VI pourrait rendre le sens progressif de l'armement] et-dans-art-dessin art-troisième art-drapeau blanc et-noir, et-dans art-parallélisme art-personnage chez-lui armes plus donc-plus 
( "Dans le troisième dessin, le drapeau est noir et blanc et, parallèlement, le personnage est de plus en plus armé »). d'indexicalité, autant au niveau lexical que syntaxique :

Wa-fî l-rasm al-thâlith al-'alam 'abyadh wa-'aswad, wa-fî l-waqt nafsi-hi [indexicalité lexicale] al-shakhs yatazâyad tasalluhu-hu [indexicalité syntaxicosémantique : utilisation du sens progressif de la forme VI yatazâyad].

et-dans art-dessin art-troisième art-drapeau blanc et-noir, et-dans art-temps (gén) même-lui art-personnage augmenter progressivement (3 p m s inac) armement (nom)-son

donc, l'arabe en tant que langue pluriglossique, peut être considérée comme une langue de spécialité quelle que soit la variété utilisée. Cette langue peut n'être que lexicalement spécialisée s'il s'agit de syntagmes dialectaux simples comme ceux utilisés dans l'activité décrite supra avec les Saint-Cyriens. Elle peut également être représentée par un propos dialectal d'une haute technicité lexicale doublé de l'insertion de syntagmes ressortissant de l'arabe classique, comme décrit supra par Dichy. Elle peut enfin être discursivement et lexicalement spécialisée, comme l'arabe classique militaire que nous enseignons aux élèves de l'EMIA.

\section{Conclusion}

La dualité de notre enseignement est induite, non pas par la réalité pluriglossique de l'usage des variétés d'arabe chez les arabophones et de leur passage de l'une à l'autre au sein d'une même situation de communication, mais par la physionomie des examens finaux requis par la sous-direction de la formation et des écoles de l'armée de Terre. Larcher («Diglossie arabisante»4) parle de « dichotomisation de son [la langue arabe] enseignement " dans la tradition didactique française. Les enseignements de l'arabe dialectal et de l'arabe classique restent donc malheureusement étanches l'un à l'autre, sauf dans les deux situations suivantes: d'une part, l'apprentissage synchronique, spontané et passif de mots et de tournures dialectales au sein du vocabulaire de la classe pour les élèves de l'EMIA apprenant l'arabe classique, apprentissage reflétant les usages des arabophones. D'autre part, le passage diachronique de l'arabe dialectal à l'arabe classique en fin de parcours d'avril à juin pour les élèves de Saint - Cyr, après que leur parcours en arabe syro - libanais est sanctionné par le CMLP1.

51 La notion de langue de spécialité et la problématique de sa distribution entre arabe dialectal et arabe classique, pose donc invariablement la problématique de l'enseignement de l'arabe en tant que langue pluriglossique. Elle pose aussi la question du niveau des apprenants: faut-il avoir un niveau élevé en arabe pour entrer dans le domaine de la langue de spécialité militaire ? S'il ne s'agit que de spécialisation lexicale et de l'activité ciblée décrite supra, l'application pédagogique de l'utilisation de l'appel militaire syrien nous montre que non. S'il s'agit de spécialisation au sens phraséologique et syntaxique du terme, au niveau discursif, l'absence d'indexicalité dans les commentaires oraux d'images en arabe classique tels que nous les avons expérimentés nous montre qu'il est nécessaire d'avoir un niveau élevé pour produire des énoncés idiomatiques. Il ne s'agit néanmoins que d'activités ciblées dont les retours d'expérience ne nous permettent pas de répondre à la question du niveau des apprenants de manière absolue et globale. Si les énoncés discursifs de nos élèves sont idiomatiques, nous pouvons

Recherche et pratiques pédagogiques en langues de spécialité, Vol. 35 № spécial 1 | 2016 
considérer que nous avons affaire à une véritable langue de spécialité militaire. Est-ce alors une question de niveau ou de pédagogie? Ce degré variable de spécialisation requiert-il une didactique spécifique? Ou doit-on reprendre les invariants de la didactique des langues étrangères en général et de la langue arabe en particulier?

Enfin, peut-on séparer notre enseignement de l'arabe de type Lansad et l'enseignement de l'arabe spécialisé militaire? Notre enseignement du dialecte, dans le contexte particulier qui est le nôtre et tel que décrit supra dans le cadre d'une activité spécifique aurait tendance, à montrer que non.

\section{BIBLIOGRAPHIE}

Cohen, David. « Koinè, langues communes et dialectes arabes. » Arabica, vol. IX, n², 1962, pp. 119-144.

Dichy, Joseph. « La pluriglossie de l'arabe. » Bulletin d'Études Orientales, Tome 46, 1994, pp. 19-42.

Dichy, Joseph. «L'enseignement de l'arabe, langue pluriglossique, dans la France d'aujourd'hui. » Les langues de la Méditerranée, volume des Cahiers de Confluences Méditerranée, dirigé par Robert Bistolfi et Henri Giordan. L'Harmattan, 2002, pp. 313-329.

Dichy, Joseph. « La pluriglossie de l'arabe en (inter)action : un exemple conversationnel syrien. » La Syrie au présent, dirigé par Baudouin Dupret et al., Actes - Sud / Sinbad, 2007, pp. 495-505.

Dressen-Hammouda, Dacia. « Langue(s) et spécialisation : deux versants d'une même problématique. " Journée d'études DILEM «Quelles réalités pour la langue de spécialité dans l'enseignement-apprentissage du LANSAD ? ", le 11 décembre 2015, Rennes I (campus Beaulieu).

Hakkak al -, Ghalib. « Les Français et les langues étrangères. Plaidoyer pour une petite révolution dans l'enseignement des langues ». al-hakkak.fr, al-hakkak.fr/PDF/

Francaisetlanguesetrangeres.pdf, rédigé le 25 janvier 2013, consulté le 2 décembre 2015.

Ibn H et al. al-Muqaddima, t. I du Kitāb al-'ibar. Maktabat al-madrasa et Dār al-kitāb al-lubnānī, 1967.

Klimiuk, Maciej. Phonetics and Phonology of Damascus Arabic. Studia Arabistyczne i slamistyczne. Monografie 1, Katedra Arabistyki i Islamistyki Uniwersytet Waszawski, 2013.

Larcher, Pierre. « Moyen arabe et arabe moyen. » Arabica, vol. XLVIII, 2001, pp. 578-609.

Larcher, Pierre. « Diglossie arabisante et fushâ vs 'âmmiyya arabes : essai d'histoire parallèle. » Selected Papers from the Eight International Conference on the History of the Language Sciences (ICHoLS VIII). Fontenay - St. Cloud, France, 14 - 19 September 1999, coll. SIHoLS, Benjamins, vol. 99, 2003, pp.47-61.

Larcher, Pierre. «Qu'est-ce que l'arabe du Coran ? Réflexions d'un linguiste. » Linguistique arabe, volume des Cahiers de linguistique de l'INALCO 5, dirigé par Georgine Ayoub et Jérôme Lentin, INALCO, 2008, pp. 27-47. 
Larcher, Pierre. «L'arabe, plus qu'une langue? » / "Is Arabic More Than Just a Language?" 54 États. Le Magazine de l'Afrique, n²4, 2015, pp. 46-49, issuu.com/priscillawolmer/docs/n__24_issuu, consulté le 2 décembre 2015.

Lelubre, Xavier. «Conception d'un dictionnaire terminologique et phraséologique trilingue anglais / français - arabe dans le domaine de l'optique. » Actualité Scientifique. Actes du Colloque de Lyon Lexicomatique et dictionnairique, Lyon, septembre 1995, bibliotheque.auf.org/doc_num.php? explnum_id=795, , consulté le 19 octobre 2016.

Lentin, Jérôme. « Damascus Arabic. » Encyclopaedia of Arabic Language and Linguistics, edited by Kees Versteegh, Brill, 2006, pp. 546-555, referenceworks.brillonline.com/entries/encyclopediaof-arabic-language-and-linguistics/damascus-arabic-EALL_COM_0077, consulté le 25 octobre 2016.

Messaoudi, Leila. « Langue spécialisée et technolecte : quelles relations ? Meta : journal des traducteurs / Meta : Translators' Journal, vol. 55, n 1, 2010, pp. 127-135.

Pinon, Catherine. « La grammaire arabe : entre théories linguistiques et applications didactiques ». Synergies Monde arabe n 2011, pp. 75-86.

Pinon, Catherine. «Les enjeux épistémologiques et didactiques d'une grammaire arabe fondée sur corpus ». Voyages grammairiens, dirigé par Teddy Arnavielle, L'Harmattan, 2012, 256 p., pp. 83-101.

Reig, Daniel. Larousse arabe, dictionnaire arabe - français. Larousse, collection « Dictionnaire maxipoche plus », 2011.

Rosenbaum, Gabriel M. « Fushâmmiyya : Alternating Style in Egyptian Prose. » Zeitschrift für Arabische Linguistik, vol. 38, Harrassowitz Verlag, 2000, pp. 68-87.

\section{NOTES}

1. Pour un état des lieux diachronique sur le rapport dialectes arabes-koinè arabe, voir Cohen 1962. Son article clarifie l'apparente synonymie entre la koinè grecque, qui n'était pas parlée, et la " 'koinè arabe' définie comme langue commune parlée dont procèderaient les dialectes " (Cohen 1962 : 119). Il confronte différentes théories sur la genèse des dialectes arabes et de l'arabe classique, avec, en toile de fond, l'hypothèse selon laquelle les dialectes citadins seraient issus d'une koinè militaire, indépendante de la koinè poétique dont serait issu l'arabe classique. Les variations sont néanmoins dues aux innovations des sédentaires, réputées plus rapides que celles des nomades (id. : 126). On ne saurait donc parler d'origine commune, mais de koinè postérieure à la genèse des dialectes. Et ce d'autant plus que ces innovations n'ont pas lieu dans tous les dialectes citadins, et ne peuvent donc pas être rattachées à la même koinè d'origine (Cohen id. :127). On ne saurait pas non plus parler d'origine "classique " commune, du fait de traits dialectaux n'existant pas dans cette variété d'arabe (id. : 143).

2. Larcher (« Diglossie arabisante »11).

3. "L'art d'écrire par abréviations", www.littre.org/definition/brachygraphie consulté le 2 décembre 2015.

4. Cet usage faisant référence aux kamikazes japonais dans le langage courant des médias occulte néanmoins le fait que ceux de la deuxième guerre mondiale appartenaient à l'armée régulière du Japon, tandis que les auteurs d'attentats-suicide dont il est question ici sont le plus souvent des combattants irréguliers ou appartenant à des organisations non reconnues.

5. Reig $2011: 488$. 
6. Pour la question de l'écart entre les grammaires arabes et arabisantes, et les usages de l'arabe contemporain, voir Pinon 2011 et 2012.

7. Décision prise par Alain Girod, chef du département de langue arabe en 2000 et actuellement Maître de Conférences à Aix-Marseille Université, avec l'accord de la hiérarchie militaire des ESCC.

8. L'utilisation de cette transcription est un pis-aller pédagogique. Nous sommes intervenue sur la question de son utilisation dans l'enseignement de l'arabe dialectal et de sa phonétique, au cours de la journée d'études DILEM «Franchir le mur des sons:la phonétique dans l'enseignement-apprentissage des langues", le 13 décembre 2013 (Rennes I, campus Beaulieu): «Les difficultés et enjeux de l'enseignement de quelques phonèmes arabes à des arabisants débutants adultes ".

9. Sur ce dialecte et ses caractéristiques, voir Lentin. Voir aussi Klimiuk.

10. Énoncés fournis par Soufiane al-Karjousli, professeur vacataire d'arabe, d'origine syrienne.

\section{RÉSUMÉS}

Cet article traite de la spécificité de l'enseignement de l'arabe aux Écoles de Saint-Cyr Coëtquidan (ESCC), école de formation initiale des officiers de l'armée de Terre française. Il montre en quoi l'enseignement de l'arabe y est particulièrement révélateur de la pluriglossie de cette langue, puisque dépendant ici des besoins des apprenants. En effet, l'arabe, en tant que langue de spécialité militaire - si nous abordons d'un point de vue lexical le concept de langue de spécialité - est étudié par deux publics différents. Cependant, étant donné que les élèves-officiers des ESCC sont considérés comme « spécialistes d'autres disciplines » et que la formation qui y est délivrée est dite « intégrée » (militaire, académique et comportementale), nous pouvons voir ici une convergence entre Lansad et langue de spécialité. Encore faut-il déterminer de quel(s) arabe (s)il s'agit.

This article deals with the specificity of Arabic language teaching in Saint-Cyr Military Academy, which trains the Cadets of the French land forces. It aims to show how Arabic language teaching reveals how much the language is pluriglossic. In fact, Cadets need different kinds of teaching in different Arabic varieties of the language. We teach Arabic in our Academy as a specialized military language (if we consider it from a lexical point of view) to two kinds of Cadets. Given that we consider them as specialist of other subjects within an embedded military, academic and behavioural training, we can see here how teaching languages to specialists of other subjects and language for specific purposes (LSP) meet. Before that, we just have to specify which sort of Arabic language we are dealing with.

classical Arabic, colloquial Arabic, literary Arabic, literal written Arabic, language for specific purposes (LSP), military language, pluriglossic environment - Saint-Cyr military Academy.

\section{INDEX}

Mots-clés : arabe classique, arabe dialectal, arabe littéraire, arabe littéral, langue de spécialité (LSP), langue militaire, pluriglossie, Saint-Cyr 


\section{AUTEUR}

\section{MARIE BAIZE-VARIN}

Marie Baize-Varin est docteur en linguistique arabe à Aix-Marseille Université, agrégée et certifiée d'arabe. Elle est maître de Conférences en linguistique arabe aux Écoles de Saint-Cyr Coëtquidan et à Rennes II. Elle est chef du département d'enseignement de l'arabe à la Direction générale de l'enseignement et de la recherche et à la Division des langues étrangères appliquées à Coëtquidan. Elle y enseigne l'arabe littéral classique, l'arabe syro-libanais et l'arabe égyptien. Chercheuse membre du Pôle d'Excellence 3 « Action globale et Forces terrestres » du Centre de recherches des Écoles de Coëtquidan, elle y est membre de l'équipe chargée de la communication. Elle est aussi chercheuse permanente à l'unité de recherche " Linguistique, ingénierie et didactique des langues » (LIDILE EA3874, Rennes I / Rennes II). Elle est également chercheuse associée à l'unité mixte de recherches « Institut de recherches et d'études sur le monde arabe et musulman » (IREMAM, UMR 7310, CNRS).

marie.varin@st-cyr.terre-net.defense.gouv.fr 